Revue de l'Institut des langues et cultures

d'Europe, Amérique, Afrique, Asie et Australie

37 | 2019

Des genres en Méditerranée : pratiques,

représentations et transfert

\title{
Les nouvelles routes de la soie et la Grèce, tête de pont de la présence chinoise en Europe
}

The Dragon's Head: Chinese Presence in Greece, Between Business and Politics

\section{Guilhem Fabre}

\section{OpenEdition}

\section{Journals}

Édition électronique

URL : http://journals.openedition.org/ilcea/7492

DOI : $10.4000 /$ ilcea.7492

ISSN : 2101-0609

Éditeur

UGA Éditions/Université Grenoble Alpes

Édition imprimée

ISBN : 978-2-37747-099-0

ISSN : 1639-6073

Référence électronique

Guilhem Fabre, «Les nouvelles routes de la soie et la Grèce, tête de pont de la présence chinoise en Europe », ILCEA [En ligne], 37 | 2019, mis en ligne le 04 novembre 2019, consulté le 10 décembre 2020. URL : http://journals.openedition.org/ilcea/7492 ; DOI : https://doi.org/10.4000/ilcea.7492

Ce document a été généré automatiquement le 10 décembre 2020.

(C) ILCEA 


\title{
Les nouvelles routes de la soie et la Grèce, tête de pont de la présence chinoise en Europe
}

The Dragon's Head: Chinese Presence in Greece, Between Business and Politics

\author{
Guilhem Fabre
}

\section{Le contexte de la nouvelle route de la soie}

1 L'arrivée au pouvoir de Xi Jinping a marqué une nette rupture non seulement dans la politique intérieure, mais aussi dans la politique extérieure chinoise, avec le lancement, puis l'élargissement progressif du projet "une ceinture, une route», (一带一路) ${ }^{1}$, abréviation de «La ceinture économique de la route de la soie, la route de la soie maritime du xxI e siècle» (丝绸之路经济带, 二十一世纪海上丝绸之路). La ceinture se réfère à la voie terrestre et ferroviaire entre l'Europe et l'Asie, tandis que la route évoque la voie maritime entre la Chine, l'Asie du Sud-Est et du Sud, l'Afrique et l'Europe.

2 Ce projet marque la fin de la diplomatie profil bas de la Chine depuis le début des réformes de 1978, qui avait été résumée par le célèbre mot d'ordre de Deng Xiaoping, « cacher son éclat et attendre son heure » (蹈光养䀲). L'heure est désormais venue. Les analyses chinoises évoquent une " période d'opportunité stratégique » jusqu'à 2020, où la Chine peut profiter d'un environnement sécuritaire bienveillant pour renforcer son pouvoir global sans causer de conflit (The Economist, 2016).

3 La ceinture économique terrestre est d'abord évoquée le 7 septembre 2013, par Xi Jingping lors d'une visite officielle au Kazakhstan, et la route maritime le mois suivant, le 3 octobre, lors d'une visite officielle en Indonésie. Le projet, désormais lancé dans toutes les enceintes internationales, et cadré le 28 mars 2015 par le document conjoint de la Commission nationale du développement et de la réforme, le ministère des Affaires étrangères et le ministère du Commerce chinois, bénéficie de financements 
prioritaires : la banque centrale a transféré 80 milliards de US $\$$ vers trois grandes banques d'État pour financer les projets OBOR, qui sont aussi soutenus par le fonds souverain chinois avec une enveloppe Route de la Soie de 40 milliards de US \$, et par l'Asian Infrastructure Investment Bank, fondée en 2016 avec un capital de 100 milliards de US \$ (The Economist, 2016).

4 En mai 2017, la tenue en Chine du « Belt and Road Forum for International Cooperation 2017 » est l'occasion d'un communiqué commun signé avec un certain nombre de chefs d'État, dont le Premier ministre grec, et d'un document sur la construction conjointe de la nouvelle route de la soie et la contribution chinoise. Ce document montre à quel point le projet Route de la soie, notamment son volet maritime, est à géométrie variable, puisqu'une centaine de pays sont signalés comme potentiellement intéressés par le projet, y compris en Amérique latine, où des accords sont signés. Une carte du projet OBOR, publiée sur le site gouvernemental chinois, montre en fait que, hormis l'Amérique du Nord, toutes les zones du monde sont potentiellement concernées. L'espace est même inclus comme dernière frontière, avec le lancement pendant l'été 2018 du projet « Route spatiale de la soie » qui vise à augmenter le développement économique de la Belt and Road Initiative (BRI) en fournissant avec Beidou des services de communication, d'imagerie et de diffusion par satellites, concurrençant à l'horizon 2020 le GPS américain, géré par l'US Air Force, le système Galileo européen et le système russe GLONASS (Hutchinson, 2018 ; Jakhar, 2018).

5 L'ensemble du projet de la route de la soie, sur terre ou maritime, concerne pour l'instant 65 pays d'Asie d'Europe et d'Afrique, représentant $29 \%$ du PIB mondial. Il s'agit essentiellement de faciliter le commerce, l'investissement et les échanges culturels dans cet ensemble. L'impact s'est déjà fait sentir puisque le commerce entre la Chine et les pays signataires a atteint 1,1 trillion de US $\$$ en 2017 , soit $15 \%$ de plus que l'année précédente (HSBC, 2017).

6 Les motivations chinoises sont autant d'ordre géopolitique, renforcer la présence et l'influence de la Chine au niveau mondial, que géoéconomique: désenclaver tout l'Ouest chinois moins développé (Xinjiang, Gansu, Tibet, Sichuan, Yunnan), diversifier ses approvisionnements énergétiques (gaz et pétrole), en Asie centrale et en Russie, exporter les compétences chinoises en matière d'infrastructures routières, ferroviaires et énergétiques, en créant de multiples corridors d'échanges (Chine-Pakistan ; YunnanSingapour); recycler les surcapacités industrielles chinoises dans les secteurs de l'acier et du ciment et exporter les standards d'innovation chinois, non seulement dans l'industrie ferroviaire et l'énergie, mais aussi dans les télécommunications, avec des sociétés en pointe comme Huawei ou ZTE, permettant d'influencer à terme, en liaison avec les majors du BAT (Baidu, Alibaba, Tencent), l'internet, le e-commerce et l'ensemble des services liés.

7 Si la route terrestre de la soie a été largement discutée (Ghiasy \& Zhou, 2017 ; Djankov \& Miner, 2016 ; Johnson, 2016 ; Amighini, 2017 ; Rolland, 2017 ; China-Britain Business Coucnil, 2015 ; Farchy \& et al., 2016) et médiatisée, avec notamment le lancement de la ligne ferroviaire Chongqing-Duisbourg, où environ $80 \%$ des trains venant de Chine font leur premier arrêt en Europe (Olterman, 2018), l'attention s'est focalisée jusqu'à présent sur la nouveauté de la voie terrestre, en analysant son impact sur l'Asie centrale (Laruelle, 2018), et en négligeant quelque peu la voie maritime. Or la voie maritime, qui part des côtes chinoises et aboutit à Athènes et Venise, est essentielle pour plusieurs raisons. 
8 En premier lieu, toute puissance globale s'est historiquement construite sur les mers, sur le plan du commerce comme sur celui de la défense. En second lieu, la part dominante du fret chinois transite par voie maritime (59\%), le fret aérien (22\%) et la route $(18 \%)$. Les voies ferrées sont en position très minoritaire (moins de $1 \%$ ), contrairement à ce qui existe en Europe $-70 \%$ du fret par route, $19 \%$ par voie maritime et $8 \%$ par voie ferrée - (Hererro \& Xu, 2016). La prépondérance du commerce maritime est encore plus importante entre l'Europe et la Chine, en termes de valeur aussi bien que de volume. Le fret aérien vient en seconde position, surtout en termes de valeur, et le rail n'occupe que la dernière place, derrière le transport routier. En troisième lieu, la stratégie chinoise, telle qu'elle est définie dans la « Vision pour une coopération maritime dans le cadre de l'initiative une ceinture une route " (Xinhua, 2017) couvre potentiellement l'ensemble des mers du globe, y compris l'Océan Arctique.

Enfin, à la différence de nombre de projets de la ceinture terrienne, au Kazakhstan ou en Croatie, qui sont encore au stade de la planification, Pékin a déjà investi massivement dans des projets de construction portuaire, de l'Asie du Sud-Est à la Méditerranée (Zand, 2018 ; Duchatel \& Duplaix, 2018).

\section{L'évolution des investissements et des prêts chinois est liée à la crise de la dette}

10 L'exemple de la Grèce est assez illustratif des enjeux et des problèmes que soulèvent la nouvelle route de la soie maritime en Méditerranée et en Europe. L'évolution des rapports sino-grecs, et des investissements chinois est en effet indissociable du contexte de la crise de la dette grecque et des plans d'austérité imposés par les bailleurs de fonds de l'Union Européenne.

11 Du point de vue de la Chine, premier exportateur mondial et numéro un de la construction navale, la Grèce a une position particulière, dans la mesure où sa marine marchande est au premier rang mondial. En 2015, les armateurs grecs contrôlaient 16 \% de la flotte mondiale de vraquiers et porte-conteneurs, et environ un quart des pétroliers géants. La flotte marchande grecque assure une large part des importations chinoises de pétrole, qui ont dépassé celle des USA, et plus de la moitié du commerce chinois de matières premières. La construction navale est un champ de coopération privilégié, dans la mesure où les commandes grecques dans les chantiers navals chinois, s'élevaient à 495 navires (2014), soit $10 \%$ des commandes mondiales (Tonchev, 2015 : 37).

12 À la fin 2017, le volume total des investissements directs étrangers (IDE) chinois en Grèce est de l'ordre de 1,2 milliards d'euros, selon le Rhodium Group et le Mercator Institute for China Studies (Haneman \& Huatori, 2018), des chiffres assez proches du ministère chinois du commerce extérieur (MOFCOM : 1,1 milliard d'euros en juin 2017) ou de l'ambassade chinoise en Grèce (1,4 milliard en octobre 2017). Mais si l'on intègre certains prêts bancaires, qui rendent mieux compte de l'influence chinoise, le stock des IDE chinois dépasserait en 2017 les 4 milliards d'euros selon une étude commandée par l'EBRD (Tonchev \& Davarinou, 2017 :14; Bastian 2017). Ceci placerait donc la Chine à la deuxième place des investisseurs étrangers en Grèce, entre l'Allemagne et la France, au lieu d'être à la dernière place du «top ten ", si l'on s'en tient aux données de la Banque de Grèce. 
13 Dans cette hypothèse, les investissements chinois en Grèce se rapprocheraient de ceux effectués au Portugal à la fin 2017. Mais indépendamment de leur volume, ce qui est le plus intéressant, dans le cas de la Grèce, est à la fois le lien étroit de ces investissements avec la crise de la dette et leur caractère stratégique.

14 En octobre 2009, un an après le début de la crise mondiale démarrée aux États-Unis, George Papandreou, le leader du Parti socialiste grec, remporte les élections législatives et révèle que la comptabilité publique grecque a été falsifiée (sur les conseils de la Banque d'affaires Goldman Sachs) et que le déficit n'est pas de $6 \%$ mais de $12,7 \%$ du PIB. La note de la Grèce chute immédiatement sur les marchés financiers internationaux, et les taux d'intérêt sur emprunts d'État s'envolent.

En avril 2010, la dette publique atteignant 35 milliards d'euros (150\% du PIB) et la politique mise en œuvre étant inopérante, la Grèce obtient une première aide de 110 milliards d'euros de l'UE et du FMI sur 3 ans, en contrepartie de mesure d'austérité drastiques : augmentation de la TVA à $23 \%$, gel du salaire des fonctionnaires, hausse des impôts, privatisations d'entreprises publiques, etc.

En octobre 2011, l'économie et les finances grecques continuent de se détériorer devant ce plan contra cyclique, avec une récession qui représente -9,1\% du PIB (Eurostat). Une deuxième tranche de prêt de 130 milliards d'euros est engagée par la zone euro, sur la base d'un effacement de $50 \%$ de la dette contractée auprès des banques privées, en échange de mesures financières encore plus strictes. Papandreou est contraint à démissionner le mois suivant. Il est remplacé par Lucas Papadémos, un économiste de la BCE qui assure l'intérim, et met en place le plan de rigueur souhaité, avec une réduction du salaire minimum de 876 à 683 euros par mois et de $12 \%$ des retraites. Le pays propose de réduire son endettement de 160 à $120 \%$ du PIB d'ici 2020, le défaut de paiement est évité.

En juin 2012, Samaras devient premier ministre d'une coalition droite-gauche, la deuxième tranche d'aide de 130 milliards d'euros est débloquée en contrepartie d'un nouveau budget d'austérité. La dette publique atteint $170 \%$ du PIB, mais la récession s'amenuise $(-7,3 \%$ du PIB en 2012, $-3,2 \%$ en $2013,-0,7 \%$ en $2014,-0,3 \%$ en 2015$)$ et le pays peut se refinancer sur les marchés financiers en 2014. En janvier 2015, Alexis Tsipras est nommé premier ministre. Issu de Syriza, une coalition de la gauche radicale, il a été élu sur la promesse de renégocier les accords de la Grèce avec ses bailleurs de fonds qui ont plongé le pays dans la plus grave crise qu'il ait connu depuis la seconde guerre mondiale : le chômage atteint 25 \% de la population active, 45 \% chez les jeunes, la récession a touché la moitié des commerces qui ont dû mettre la clé sous la porte, les coupes de salaire, de retraites, et de dépenses publiques ont provoqué une crise humanitaire dans un pays où les aides sociales sont pratiquement inexistantes.

Les négociations avec l'UE et les créanciers aboutissent à un plan d'aide dont les conditions sont soumises à un référendum fin juin 2015 (61\% de «non»), mais alors qu'on évoque de plus en plus le risque de "Grexit» la renégociation de la dette lors d'un sommet de chefs d'États européens le 13 juillet 2015, débouche sur le principe d'un troisième plan d'aide de 86 milliards d'euros, en échange d'un retournement de la politique de Syriza, d'une réforme des retraites allongeant la durée des cotisations, et du lancement d'une politique de privatisation massive des actifs publics sous l'égide de la Hellenic Republic Asset Developement Fund (HRADF), accompagnant plus d'une centaine de mesures de rigueur officiellement intitulées "programme d'ajustement macro-économique » (Tragranides, 2018: 56-59 \& Tonchev, $2017: 27$ ). 

avec l'Union Européenne et les bailleurs de fonds que se développe petit à petit un partenariat sino-grec. Au début de la crise de la dette, la Chine, sollicitée par le premier ministre George Papandreou, ancien président de l'internationale socialiste, a investi 6 milliards d'euros dans la dette publique grecque. Cinq ans plus tard au début 2015, le premier ministre Alexis Tsipras s'attendait à ce que la Chine s'engage sur un prêt important pour alléger la dette publique, mais il n'obtint de Pékin, qu'un achat très prudent de 100 millions de US $\$$ de bons du trésor (Tonchev, 2017 : 49 \& Tzogopoulos, 2015). effet de diminuer massivement l'épargne et l'investissement (OCDE, 2017). Dans un contexte de chute très importante du prix des actifs, privés ou publics, l'investissement étranger prend le relais, en bénéficiant de cette opportunité d'affaires sans précédent. Les actifs publics sont les premiers concernés par les politiques de privatisation, devant le déficit abyssal. C'est dans ce contexte de grande vulnérabilité de l'économie et de la société grecque qu'interviennent les IDE chinois.

\section{3. "La tête du dragon » ou l'investissement chinois phare dans le port du Pirée}

21 En 2009, en dépit de l'opposition très forte des syndicats de dockers et d'une partie importante de la classe politique grecque, la compagnie $\mathrm{COSCO}$, troisième transporteur maritime mondial (après APM Maersk et Mediteranean Shg Co.) obtient à la suite de longues négociations, la gestion des terminaux II et III du port de conteneur du Pirée, le quai I restant géré par la compagnie d'État, Piraeus Port Authority (PPA) pour un paiement initial de 50 millions d'euros, et des investissements importants visant à augmenter les capacités du port.

22 En 2016, dans le nouveau contexte du programme de privatisation accéléré imposé par les bailleurs de fonds européens, COSCO signe un accord qui lui donne $51 \%$ des parts du PPA, pour 280,5 millions d'euros, et à terme $16 \%$ de plus de parts d'ici 2021 , si cosCO investit 350 millions d'euros dans le port d'ici cette date et verse un complément de 88 millions d'euros.

23 Le but de COSCO est dans un premier temps de transformer le port du Pirée en tête de pont du commerce chinois vers l'Europe du Sud et de l'Est, et en plate-forme de transbordement vers les autres ports de la Méditerranée orientale, et dans un deuxième temps depuis 2016, en port d'attache de l'industrie des croisières en Méditerranée, capable d'accueillir 14 navires de croisière à la fois, dont quatre géants, et plusieurs millions de touristes par an. COSCO envisage aussi des docks de réparation navale pour des navires jaugeant plus de 80000 tonnes (Tonchev, $2017: 16-17$; Van der Putten, 2014 ; Psaraftis \& Pallis, 2012 : 27-43).

La taille des porte-conteneurs de plus en plus grande crée en effet une demande de transbordement pour des navires plus réduits. L'intérêt de la Méditerranée pour le transport maritime global s'est accentué avec l'élargissement du canal de Suez en 2015, qui a permis de doubler la circulation des navires sur 72 de ses $193 \mathrm{~km}$ avec l'élargissement de $37 \mathrm{~km}$ de voie initiale et le creusement d'une nouvelle voie sur $35 \mathrm{~km}$. La réduction du temps de traversée est de 20 à $11 \mathrm{~h}$ dans un sens et de 8 à $3 \mathrm{~h}$

ILCEA, 37 | 2019 
dans l'autre, et l'on s'attend à un doublement du trafic entre 2015 et 2024. Ces travaux s'inscrivaient eux-mêmes dans un contexte de décuplement des exportations chinoises vers l'UE, de 33 à 338 milliards entre 1999 et 2016. La Méditerranée représenterait environ $35 \%$ des flux maritimes de conteneurs chinois vers l'Europe (Pairault, 2019), et cette tendance a toute chance de s'accentuer en fonction des économies de transport réalisées. Un conteneur chargé à Shanghai coûte environ 100 \$ de moins s'il est destiné à un port de Méditerranée par rapport à un port d'Europe du Nord (Duchatel \& Duplaix, $2018: 10)$, le temps de transport est réduit d'une semaine, et l'économie pour un porteconteneur est estimée au moins à 2 millions de US \$ (Psaropoulos, 2017).

Mais l'intérêt de la Méditerranée ne se réduit pas à ce corridor maritime entre la Chine et l'Europe. Les porte-conteneurs géants sur le trajet entre la Chine et la côte Est des États-Unis empruntent de plus en plus le canal de Suez, le canal de Panama n'étant pas adapté, en dépit de ses travaux d'élargissement, à la taille considérable de ces navires. On assiste donc à un basculement de la route transpacifique vers la route méditerranéenne et transatlantique pour le trafic de conteneurs entre l'Asie et la côte Est de l'Amérique du Nord. En 2014, la capacité de conteneur d'Asie vers la côte nordaméricaine à travers le canal de Suez a dépassé pour la première fois celle du canal de Panama (Van der Putten \& Meijnders, 2015 : 29).

La Méditerranée a ainsi attiré, indépendamment de Cosco, plusieurs grandes compagnies chinoises de transport maritime, qui ont acquis la gestion de terminaux portuaires (Pairault, 2019). Mais le port du Pirée, acquis en majorité par COSCO constitue un projet phare à plus d'un titre. D'abord parce que COSCO a introduit les équipements les plus modernes, permettant une relance très importante de la productivité du travail, dans un port où le salaire des dockers atteignait des dimensions rentières en 2004, avec plus de 140000 euros par an pour 325 à 335 jours ouvrés (Psaraftis \& Pallis, 2012 : 42-43). En second lieu parce que cosco est lui-même un des principaux acteurs du transport maritime Chine-Europe et à ce titre un utilisateur privilégié du port, entrainant dans son sillage K-Line, Hanjin et Yang Ming, ses partenaires de la Green Alliance, et les autres géants du transport maritime, comme APM-Maersk et CMA-CGM. Enfin parce que COSCO a su attirer des compagnies internationales importantes, comme Hewlett-Packard, qui ont relocalisé une partie importante de leurs activités de distribution de Rotterdam au Pirée, sans parler des projets de multinationales comme Huawei, ZTE, Lenovo, Dell, IKEA et LG (Van der Putten, 2014 : 16-17).

Les résultats sont là, alors qu'au début des années 2010, le transbordement de conteneurs était supérieur au Pirée dans la plupart des ports méditerranéens concurrents (Port-Saïd et Damietta en Égypte), Algeciras et Valence en Espagne, Gioia Tauro en Italie, Tanger au Maroc, Malte et finalement Ambarli en Turquie, dès 2013, le port du Pirée se place en troisième position du trafic de conteneur en Méditerranée, derrière Algeciras et Valence, et devant Port-Saïd, et à la $41^{\mathrm{e}}$ place au niveau mondial (Van der Putten, 2014: 14 \& World Shipping Council, 2019). L'ambition affichée de CosCO est de monter à un trafic de 7 millions de conteneurs Équivalent Vingt Pieds (EPV) d'ici 2022, en faisant du Pirée le $5^{\mathrm{e}}$ port d'Europe, avec des investissements annoncés de l'ordre de 5 milliards d'US $\$$ dans les prochaines années, dont une bonne partie sera consacrée à des hôtels et à des centres commerciaux au sein du port (Tonchev, $2017: 17-18,24$ ). 


\section{d}

de l'équipement de télécoms, des téléphones mobiles, et investit dans la création de centres de R\&D, tandis que ZTE tout en fournissant des équipements, propose aussi des services de téléphonie, une gestion du big data, et se positionne par rapport au développement du prochain réseau 5G. Enfin Pacific Century CyberWorks (PCCW), une filiale de Hongkong Telecom, se situe sur le même créneau que ZTE, tout en montrant son intérêt dans la construction d'une infrastructure internet haut débit en liaison avec la Telecommunication Organisation of Greece - OTE (Tonchev \& Davarinou, 2017 : 17-19, 50 ; Wakim, 2018). 

et 2017, le groupe Fosun participe à hauteur de 200 millions d'euros au consortium grec pour le développement de l'ancien aéroport d'Hellenikon, un projet de 6,2 millions de $\mathrm{m}^{2}$ avec $3,5 \mathrm{~km}$ de côtes. 850 citoyens chinois ont investi dans des propriétés d'en moyenne 550000 à 600000 euros pour bénéficier du Golden Visa programme, qui leur donne un droit de résidence automatique. Enfin les perspectives du tourisme chinois en Grèce sont très prometteuses, avec la création de deux lignes aériennes directes entre la Chine et Athènes avec Air China et China Eastern Airlines, amenant les masses de touristes attendus dans les navires de croisière basés au Pirée, en partance pour la mer Égée, la Méditerranée et la côte adriatique.

La plupart des investissements chinois sont le fait d'entreprises d'État supervisées par le pouvoir politique, et bénéficient des lignes de crédit prioritaires de la China Development Bank, qui collabore étroitement avec la banque centrale de Grèce et le Hellenic Republic Asset Development Fund chargé des privatisations (Tonchev \& Davarinou, $2017: 20-26,40$ ). Les visites successives du premier ministre chinois et du Président Xi Jinping en Grèce en 2014, montrent l'intérêt des plus hautes autorités pour ce pays en tant que tête de pont des IDE chinois. On assiste bien à la mise en place d'une ambitieuse politique d'investissement couvrant trois secteurs décisifs (port, énergie, télécommunications). Cette présence est certes utile à la Grèce dans le cadre de la baisse générale des investissements intérieurs, mais elle s'accompagne d'un déséquilibre important des échanges et plus subtilement d'une politique d'influence politique, dont les contours se préciseront au fil du temps.

\section{De l'asymétrie économique aux dividendes politiques}

L'asymétrie économique entre la Chine et la Grèce est inscrite dès le début des rapports entre les deux pays. Avec une population d'une dizaine de millions d'habitants et un PIB de 268 milliards de US $\$$ en parité de pouvoir d'achat, la Grèce est comparable économiquement à une seule municipalité chinoise, comme celle de Hangzhou :

ILCEA, 37 | 2019 


\begin{tabular}{|c|c|c|c|}
\hline & \multirow[t]{2}{*}{ Grèce } & \multicolumn{2}{|c|}{ Grèce en $\%$ du } \\
\hline & & $\begin{array}{c}\text { Europe- } \\
\text { CEI }\end{array}$ & Monde \\
\hline Population (milliers) & 10771 & 1,18 & 0,14 \\
\hline $\begin{array}{l}\text { PIB aux prix courants } \\
\text { (millions de } S \text { courants) }\end{array}$ & $\begin{array}{l}192 \\
691\end{array}$ & 0,95 & 0,25 \\
\hline $\begin{array}{l}\text { PIB PPA (millions de } \$ \\
\text { constants PPA) }\end{array}$ & $\begin{array}{l}268 \\
427\end{array}$ & 1,01 & 0,24 \\
\hline $\begin{array}{l}\text { PIB par tête (\$ } \\
\text { courants) }\end{array}$ & 17891 & 80,44 & 175,23 \\
\hline $\begin{array}{l}\text { PIB par tête en PPA ( } \$ \\
\& \text { prix constants) }\end{array}$ & 24922 & 85,42 & 165,60 \\
\hline \multicolumn{4}{|c|}{ Commerce (millions de dollars courants) } \\
\hline Exportations de biens & 27811 & 0,45 & 0,18 \\
\hline $\begin{array}{l}\text { Exportations de } \\
\text { services }\end{array}$ & 27754 & 1,20 & 0,59 \\
\hline
\end{tabular}

Source : Données CEPII, 2017.

Le poids commercial de la Chine n'a fait que se renforcer, passant entre 2006 et 2016, de la $9^{\mathrm{e}}$ place à la $3^{\mathrm{e}}$ place des importations grecques, même si cette tendance s'est un peu modifiée en 2017.

\section{Grèce - Part des 20 premiers partenaires dans les exportations et les importations 0}

en \% des exportations ou importations de biens, 2006 et 2016

\begin{tabular}{|c|c|c|c|c|c|c|c|}
\hline Exportations & 2006 & Exportations & 2016 & Importations & 2006 & Importations & 2016 \\
\hline Italie & 11,3 & Italie & 10,7 & Allemagne & 12,3 & Allemagne & 10,9 \\
\hline Allemagne & 10,5 & Allemagne & 7,3 & Italie & 11,5 & Italie & 8,4 \\
\hline Royaume-Uni & 5,8 & Chypre & 5,3 & Russie & 6,2 & Chine & 7,1 \\
\hline Chypre & 5,8 & Turquie & 5,1 & France & 6,1 & Russie & 6,3 \\
\hline Bulgarie & 5,6 & Etats-Unis & 4,9 & Pays-Bas & 5,1 & Pays-Bas & 5,5 \\
\hline Turquie & 5,0 & Bulgarie & 4,8 & Corée du Sud & 4,2 & Irak & 5,0 \\
\hline Etats-Unis & 4,8 & Royaume-Uni & 4,4 & Belgique & 4,1 & France & 4,4 \\
\hline France & 4,5 & Liban & 4,1 & Arabie saoudite & 3,8 & Corée du Sud & 4,2 \\
\hline Espagne & 4,0 & Egypte & 3,2 & Chine & 3,8 & Belgique & 3,8 \\
\hline Roumanie & 3,1 & Roumanie & 3,2 & Royaume-Uni & 3,8 & Espagne & 3,7 \\
\hline Albanie & 2,2 & France & 2,8 & Espagne & 3,7 & Bulgarie & 3,5 \\
\hline Pays-Bas & 2,1 & Espagne & 2.4 & Iran & 3,3 & Turquie & 3,2 \\
\hline
\end{tabular}

Source : CEPII 
Les principaux fournisseurs de la Grèce en 2017

\begin{tabular}{|c|c|c|c|c|c|}
\hline En M€ & $\begin{array}{l}\text { Importations } \\
2017\left(^{\star}\right)\end{array}$ & $\begin{array}{c}\Delta 2017 / 2016 \\
(\%)\end{array}$ & $\begin{array}{l}\text { Part import } \\
2017(\%)\end{array}$ & $\begin{array}{l}\text { Part import } \\
2016(\%)\end{array}$ & $\begin{array}{l}\text { Class. } \\
2017\end{array}$ \\
\hline Allemagne & $5.041,3$ & $+6,7 \%$ & $10,3 \%$ & $11,0 \%$ & $1(=)$ \\
\hline Italie & $3.735,6$ & $+4,1 \%$ & $7,6 \%$ & $8,3 \%$ & $2(=)$ \\
\hline Russie & $3.421,2$ & $+21,2 \%$ & $7,0 \%$ & $6,5 \%$ & $3(+1)$ \\
\hline Corée du Sud & $3.163,1$ & $+76,8 \%$ & $6,5 \%$ & $4,1 \%$ & $4(+4)$ \\
\hline Irak & $3.148,3$ & $+34,2 \%$ & $6,4 \%$ & $5,4 \%$ & $5(=)$ \\
\hline Chine & $2.719,1$ & $-5,8 \%$ & $5,5 \%$ & $6,7 \%$ & $6(-3)$ \\
\hline
\end{tabular}

Source : France Trésor.

Mais ce qui est frappant, c'est le déséquilibre massif des échanges, qui fait que la Grèce importe de Chine dix fois plus qu'elle n'exporte :

Greece's International Trade in Goods with China (in $€$ billion) 2013 - 2015

\begin{tabular}{|c|c|c|c|}
\hline & Exports & Imports & Balance \\
\hline $\mathbf{2 0 1 3}$ & 0.419 & 2.19 & -1.78 \\
\hline $\mathbf{2 0 1 4}$ & 0.279 & 2.49 & -2.21 \\
\hline $\mathbf{2 0 1 5}$ & 0.271 & 2.87 & -2.67 \\
\hline
\end{tabular}

Sources: For 2013 and 2014 data: Eurostat newsrelaease: 116/2015 26. June 2015 http://ec.europa.eu/eurostat/documents/2995521/6893875/6-26062015-AP-EN.pdf/44d4c87c-98dd-4061-bdf6b292884a5073. For 2015 data see: http://atlas.media.mit.edu/en/profile/country/gred.

Cette situation n'est pas prête de se modifier, la Grèce servant surtout de tête de pont pour la Chine au niveau de ses exportations dans le cadre de la BRI. La Chine n'est toujours pas présente dans les principaux débouchés extérieurs de la Grèce en 2017 : 
Les principaux clients de la Grèce en 2017

\begin{tabular}{|c|c|c|c|c|c|}
\hline En M€ & $\begin{array}{l}\text { Exportations } \\
\qquad 2017\left(^{*}\right)\end{array}$ & $\begin{array}{c}\Delta 2017 / 2016 \\
(\%)\end{array}$ & $\begin{array}{l}\text { Part export } \\
2017(\%)\end{array}$ & $\begin{array}{l}\text { Part Export } \\
2016(\%)\end{array}$ & $\begin{array}{l}\text { Class. } \\
2017\end{array}$ \\
\hline Italie & $3.037,8$ & $+8,1 \%$ & $10,7 \%$ & $11,2 \%$ & $1(=)$ \\
\hline Allemagne & $2.015,5$ & $+4,4 \%$ & $7,1 \%$ & $7,7 \%$ & $2(=)$ \\
\hline Turquie & $1.951,5$ & $+44,4 \%$ & $6,9 \%$ & $5,4 \%$ & $3(+1$ \\
\hline Chypre & $1.724,5$ & $+13,2 \%$ & $6,1 \%$ & $6,1 \%$ & $4(-1)$ \\
\hline Bulgarie & $1.348,6$ & $+7,7 \%$ & $4,7 \%$ & $5,0 \%$ & $5(=)$ \\
\hline Liban & $1.249,3$ & $+19,1 \%$ & $4,4 \%$ & $4,2 \%$ & $6(+2)$ \\
\hline
\end{tabular}

Source : France Trésor.

La seule solution existante pour rétablir l'équilibre des échanges est d'avoir recours, indépendamment du transport maritime, au tourisme chinois, activité exportatrice de services par excellence, qui permet généralement d'équilibrer la balance courante de la Grèce, mais crée en même temps un effet de vulnérabilité en cas d'instabilité politique ou sociale, comme l'Égypte en montre l'exemple. 8 à $10 \%$ des touristes chinois en Grèce sont des acheteurs potentiels de propriété immobilière (Tonchev \& Davarinou, 2017 : $34)$.

Balance commerciale de la Grèce 2011-2017

\begin{tabular}{lcccccccc} 
En Mds & 2011 & 2012 & 2013 & 2014 & 2015 & $\begin{array}{c}2016 \\
(*)\end{array}$ & $\begin{array}{c}2017 \\
(*)\end{array}$ & $\begin{array}{c}\Delta \\
\mathbf{2 0 1 7 / 2 0 1 6}\end{array}$ \\
\hline Exportations & 24,38 & 27,58 & 27,30 & 27,12 & 25,48 & 25,15 & 28,46 & $+13,2 \%$ \\
\hline Importations & 48,89 & 49,54 & 47,00 & 48,33 & 42,65 & 43,12 & 49,03 & $+13,7 \%$ \\
\hline Total & 73,27 & 77,12 & 74,30 & 75,45 & 68,13 & 68,27 & 77,49 & $+13,5 \%$ \\
\hline Déficit & $-24,51$ & $-21,96$ & $-19,70$ & $-21,21$ & $-17,17$ & $-17,97$ & $-20,56$ & $+14,4 \%$ \\
\hline $\begin{array}{l}\text { Taux de } \\
\text { couverture }\end{array}$ & $49,9 \%$ & $55,7 \%$ & $58,1 \%$ & $56,1 \%$ & $59,7 \%$ & $58,3 \%$ & $58,0 \%$ & \\
\hline
\end{tabular}

(\%) 
Evolution des principaux agrégats de l'économie en \% du PIB

(\% variations annuelles, prix constants)

\begin{tabular}{lcccccccc} 
& $\mathbf{2 0 1 5}$ & $\mathbf{2 0 1 6}$ & $\mathbf{2 0 1 7}$ & $\mathbf{2 0 1 8}$ & $\mathbf{2 0 1 9}$ & $\mathbf{2 0 2 0}$ & $\mathbf{2 0 2 1}$ & $\mathbf{2 0 2 2}$ \\
\hline PIB & $-0,3 \%$ & $-0,2 \%$ & $1,4 \%$ & $2,0 \%$ & $2,4 \%$ & $2,3 \%$ & $2,1 \%$ & $1,8 \%$ \\
\hline Consommation privée & $-0,5 \%$ & $0,0 \%$ & $0,1 \%$ & $0,5 \%$ & $1,0 \%$ & $1,2 \%$ & $1,2 \%$ & $1,2 \%$ \\
\hline Consommation publique & $1,2 \%$ & $-1,5 \%$ & $-1,1 \%$ & $1,2 \%$ & $0,4 \%$ & $0,6 \%$ & $0,4 \%$ & $0,4 \%$ \\
\hline $\begin{array}{l}\text { Formation brute de } \\
\text { capital fixe }\end{array}$ & $-0,3 \%$ & $1,6 \%$ & $9,6 \%$ & $11,1 \%$ & $12,1 \%$ & $9,4 \%$ & $7,7 \%$ & $5,7 \%$ \\
\hline $\begin{array}{l}\text { Exportations biens et } \\
\text { services }\end{array}$ & $3,1 \%$ & $-1,8 \%$ & $6,8 \%$ & $5,6 \%$ & $4,6 \%$ & $4,4 \%$ & $3,0 \%$ & $3,0 \%$ \\
\hline $\begin{array}{l}\text { Importations biens et } \\
\text { services }\end{array}$ & $0,4 \%$ & $0,3 \%$ & $7,2 \%$ & $5,5 \%$ & $4,4 \%$ & $4,2 \%$ & $2,9 \%$ & $2,9 \%$ \\
\hline
\end{tabular}

Source : France Trésor.

Consciente de ce déséquilibre et de la vulnérabilité particulière de la Grèce en Europe, prisonnière d'une dette encore très importante dans les années à venir (150\% du PIB à l'horizon 2022), la Chine a rehaussé la Grèce au rang de partenaire d'égal à égal au niveau diplomatique. Indépendamment de la visite de ses plus hauts dirigeants, la Grèce a été l'objet d'une attention particulière au niveau des relations culturelles, avec le lancement du «Ancient Civilisations Forum » à Athènes, plus connu sous le nom de GC 10, qui regroupe aux cotés de la Chine et de la Grèce, la Bolivie, l'Égypte, l'Inde, l'Irak, l'Italie, le Mexique et le Pérou. «La Grèce est l'amie la plus sûre de la Chine en Europe ", assure le Président Xi Jinping en recevant à Pékin le premier ministre Alexis Tsipras en juillet 2016. Les enquêtes d'opinion montrent que la popularité de la Chine vient juste après celle de la Russie en Grèce, devant les États-Unis, et l'Allemagne, plutôt décriée (Tonchev \& Davarinou, $2017: 35,42,52)$. Bien que les Grecs soient conscients des enjeux difficiles que représente la montée de la Chine par rapport à l'Europe, ou des défis qu'elle pose dans sa pratique en matière de droits de l'homme, d'environnement, ou de forme de gouvernement, la grande majorité d'entre eux $(83,5 \%)$ est en faveur d'une coopération économique renforcée, et même de liens politiques plus étroits ( $71 \%$ ) dont ils attendent une relance de l'emploi local (Tonchev, $2018: 36-37)$ 
Evolution de la dette du gouvernement général (en M€)

\begin{tabular}{l|r|r|r|r|r|r|r}
\hline & 2016 & 2017 & 2018 & 2019 & 2020 & 2021 & 2022 \\
\hline Dette & 315.009 & 317.407 & 335.000 & 323.300 & 318.300 & 313.300 & 318.700 \\
\hline $\begin{array}{l}\text { en \% du } \\
\text { PIB }\end{array}$ & $180,8 \%$ & $178,6 \%$ & $183,1 \%$ & $170,4 \%$ & $161,4 \%$ & $153,1 \%$ & $150,3 \%$ \\
\hline $\begin{array}{l}\text { Taux } \\
\text { unifiés }\end{array}$ & 5.615 & 5.626 & 6.208 & 6.295 & 6.392 & \\
\hline $\begin{array}{l}\text { en \% du } \\
\text { PIB }\end{array}$ & $3,2 \%$ & $3,2 \%$ & $3,4 \%$ & $3,3 \%$ & $3,2 \%$ & $3,3 \%$ & $3,3 \%$ \\
\hline $\begin{array}{l}\text { PIB } \\
\text { Source : France Trésor. }\end{array}$ & 174.199 & 177.735 & 182.959 & 189.743 & 197.218 & 204.572 & 212.002 \\
\hline
\end{tabular}

41 Le soft power chinois est une priorité de Xi Jinping, dans le cadre de sa nouvelle politique d'affirmation internationale. Les partenariats avec les médias étrangers sont passés d'une soixantaine en 2000 à 221 en 2017, visant particulièrement les 60 millions de chinois vivant à l'étranger. De nombreuses personnalités politiques conseillent les entreprises chinoises ou facilitent les rapports sino-européens, dont David Cameron, responsable d'un fond sino-britannique de 1 milliard de US \$ soutenant les investissements d'infrastructure de la BIR, Jean-Pierre Raffarin, Dominique de Villepin, Romano Prodi, etc. (Feng, 2018 ; Crawford et al., 2018)

Les dividendes politiques de la présence chinoise en Grèce sont impressionnants dès 2016. En premier lieu, la Grèce s'oppose pour la première fois en juillet 2016, à une déclaration conjointe de l'Europe sur la dispute concernant la Mer de Chine du Sud, où un chapelet d'îles annexées par la chine font l'objet d'installations militaires, contraires au droit international de la mer. La Hongrie et la Croatie, membres de l'Union Européenne, rejoignent la Grèce sur cette position qui empêche l'Europe de parler d'une seule voix. La Serbie, non membre de l'UE, soutient aussi la position chinoise. L'année suivante, en juin 2017, une résolution européenne sur le bilan des droits de l'homme en Chine, est bloquée par la Grèce au Conseil des Nations unies sur les droits de l'homme, à Genève, ce qui empêche son adoption. Le Global Times, ou la voix de Pékin, déclare aussitôt que « contrairement à l'Union européenne, qui a traité la Grèce comme un emprunteur délinquant, Beijing considère le pays comme un partenaire sûr » (Tonchev \& Davarinou, 2017 : 53).

43 Au nom de l'amitié avec la Chine, une brèche est donc ouverte au sein des valeurs défendues par l'Europe des États, sans qu'on sache encore jusqu'où conduiront les interactions subtiles entre présence économique et influence politique de la Chine. Tout au plus peut-on noter certains faits troublants : la marine chinoise a effectué de nombreuses visites au Pirée, d'habitude à la fin de patrouilles anti-piraterie effectuées dans le Golfe d'Aden, ce qui a relancé les spéculations sur un rôle dual de l'investissement de cosco. En effet en juin 2015, le gouvernement chinois a annoncé 
qu'à l'avenir les constructeurs de navires chinois civils devaient s'assurer que ceux-ci puissent être compatibles avec une utilisation militaire en cas d'urgence. Cette nouvelle stratégie de conversion de la flotte civile en force militaire vise à la protection des lignes stratégiques et de communication et de transports maritimes (Tonchev \& Davarinou, 2017 : 55-56 ; Zhao, 2015).

Signe de temps contradictoires : d'un coté, en préparation du sommet UE-Chine, qui s'est tenu en juillet 2018, 27 des 28 ambassadeurs de l'UE à Pékin ont critiqué dans un rapport interne la BRI, en estimant qu'elle allait contre l'agenda de libéralisation du commerce de l'UE et déstabilisait l'équilibre du pouvoir en faveur d'entreprises chinoises subventionnées (Heide et al., 2018). Mais de l'autre, la Grèce est le premier pays occidental à signer avec la Chine, en août 2018, un " protocole d'entente » sur la BRI (Xinhua, 2018).

Quels que soient les bénéfices indéniables de la présence chinoise en Grèce, il est donc important d'être attentif à l'utilisation politique qui peut en être faite par le gouvernement chinois au sein de l'Union européenne, afin que la « tête du dragon » ne mine pas progressivement, entre les interventions de la Grèce et celles de la Hongrie, le fragile équilibre des valeurs dont se réclame l'Union.

\section{BIBLIOGRAPHIE}

AMIGHINI Alessia [dir] (2017), China's Belt and Road: A game changer?, Milan : ISPI (Italian Institute for International Policy Studies).

BASTIAN Jens (2017), «The potential for growth through Chinese infrastructure investments in Central and South Eastern Europe along the Balkan Silk Road », en ligne sur European Bank for Reconstruction and Development (EBRD) : <https://www.ebrd.com/news/2017/what-chinas-beltand-road-initiative-means-for-the-western-balkans.html> (9 janvier 2019).

Belt AND ROAD Forum For InTERNATIONAL COOPERATION (2017), « Joint Communique of the Leaders Roundtable of the Belt and Road Forum for International Cooperation », en ligne sur Belt and Road Portal : <http://www.beltandroadforum.org/english/n100/2017/0516/c22-423.html> et <http:// www.xinhuanet.com/politics/2017-05/10/c_1120951928.htm> (8 janvier 2019).

BELT AND ROAD PORTAL (Portail de la nouvelle route de la soie), en ligne sur <https:// eng.yidaiyilu.gov.cn/index.htm> (8 janvier 2019).

ChInA-BRITAIn Business Council (2015), One Belt, One Road: A Role for UK Companies in Developing China's New Initiative, Londres.

COMMISSION NATIONALE dU DÉVELOPPEMENT ET DE LA RÉFORME, MinisTère DES AFFAIRES ÉTRANGÈRES DE LA RPC, MinistÈre DU COMMERCE DE LA RPC (2015), Promote the Construction of the Silk Road Economic Belt and the Vision and Action of the Maritime Silk Road in Twenty-first Century, en ligne sur <https:// baike.baidu.com/item/推动共建丝绸之路经济带和21世纪海上丝绸之路的愿景与行动/ 17010381> (document en chinois, 28 mars 2015 ; 8 janvier 2019). 
CRAWFORD Alan et al. (2018), « Riding China's Rise: the European Politicians in Beijing's Orbit », en ligne sur Bloomberg : <https://www.bloomberg.com/news/articles/2018-07-03/riding-china-srise-the-european-politicians-in-beijing-s-orbit>.

DJANKov Simeon \& MinER Sean (2016), China's Belt and Road Initiative: Motives, Scope, and Challenges, Washington : Peterson Institute for International Economics.

Duchatel Mathieu \& DuPLAIX Alexandre Sheldon (2018), « Blue China: Navigating the Maritime Silk Road to Europe », en ligne sur European Council of Foreign Relations Policy Brief : <www.ecfr.eu> (8 janvier 2019).

FARCHY Jack et al. (2016), « One belt, one road: a ribbon of road, rail and energy projects to help increase trade », en ligne sur Financial Times : <https://ig.ft.com/sites/special-reports/one-beltone-road/> (8 janvier 2019).

FENG Emily (2018), « China and the world: how Beijing spreads the message », en ligne sur Financial Times : <https://www.ft.com/content/f5d00a86-3296-11e8-b5bf-23cb17fd1498> (8 janvier 2019).

GHIASY Richard \& ZHOU Jiayi (2017) The Silk Road Economic Belt: Considering security implications and EU-China cooperation prospects, Stockholm : Stockholm International Peace Research Institute (SIPRI) and Friedrich Ebert Stiftung.

HANEMANN Thilo \& HUATORI Mikko (2018), « EU-China FDI: Working towards reciprocity in investment relations », en ligne sur Mercator Institute for China Studies : <https://www.merics.org/ en/papers-on-china/reciprocity> (8 janvier 2019).

HEIDE Dana et al. (2018), « EU ambassadors band together against Silk Road », Handelsblatt Global (6).

HERERRO GARCIA Alice \& XU Jinawei (2016), China's Belt and Road initiative: Can Europe expect trade gains?, Working Paper, Issue 5, Bruxelles : Bruegel.

HSBC (2018), The Belt and Road Initiative, en ligne sur <https://www.business.hsbc.com/en-gb/ belt-and-road> (8 janvier 2019).

HUTCHINSON Ian (2018), « Belt and Road: The Final Frontier », en ligne sur China Business Review : $<$ http://www.chinabusinessreview.com/belt-and-road-the-final-frontier/> (8 janvier 2019).

JAKHAR Pratik (2018), « How China's GPS 'rival' Beidou is plotting to go global », en ligne sur BBC News : <https://www.bbc.com/news/technology-45471959> (8 janvier 2019).

JoHnson Christopher K. (2016), « President Xi Jinping's “Belt and Road” Initiative: A Practical Assessment of the Chinese Communist Party's Roadmap for China's Global Resurgence », en ligne sur Center for Strategic \& International Studies : <https://www.csis.org/analysis/president-xijinping\%E2\%80\%99s-belt-and-road-initiative>.

LARUELLE Marlene [dir.] (2018), China's Belt and Road Initiative and its Impact in Central Asia, George Washington University Central Asia Program : Washington.

OCDE (2017), « Profil statistique de la Grèce, 2008-2015 », en ligne sur OECD iLibrary : <https:// www.oecd-ilibrary.org/economics/profil-statistique-par-pays-grece-2017-1_csp-grctable-2017-1-fr> (9 janvier 2019).

OlteRMAN Philip (2018), « Germany's “China city”: How Duisburg became Xi Jinping's gateway to Europe », en ligne sur The Guardian : <https://www.theguardian.com/cities/2018/aug/01/ germanys-china-city-duisburg-became-xi-jinping-gateway-europe> (8 janvier 2019). 
PAIRAULT Thierry (2019), « La route maritime de la soie et les terminaux portuaires en Méditerranée ", Monde chinois, à paraître.

Rolland Nadège (2017), China's Eurasian Century? Political and Strategic Implications of the Belt and Road Initiative, The National Bureau of Asian Research Books : Seattle.

PSARAfTIS Harilaos N. \& PALLIS Athanasios A. (2012), « Concession of the Piraeus container terminal: turbulent times and the quest for competitiveness ", Maritime Policy \& Management: The flagship journal of international shipping and port research, 39(1), 27-43, <http://martrans.org/ documents/2012/prt/MPM2012-Psaraftis.pdf> (9 janvier 2019).

PSAROPOUlOS John (2017), « Greece's Pivot toward China », en ligne sur The New Athenian : <http:// www.thenewathenian.com/2017/07/greeces-pivot-toward-china.html> (9 janvier 2019).

StANZEL Angela et al. (2016), "China's investment in influence: the future of $16+1$ cooperation », en ligne sur European Council on Foreign Relations : <https://www.ecfr.eu/publications/summary/ chinas_investment_in_influence_the_future_of_161_cooperation7204> (9 janvier 2019).

THE ECONOMIST (2016), Our bulldozers, our rules: China's foreign policy could reshape a good part of the world economy, en ligne sur <https://www.economist.com/china/2016/07/02/our-bulldozers-ourrules>.

ToNCHEv Plamen (2015), « Greece and China: Discovering each other at last? », M. U. Uatori et al. (dir.), Mapping Europe-China Relations, A Bottom-Up Approach, European Think Tank Network on China (ETNC), <http://www.iai.it/sites/default/files/2015_etnc_report.pdf>.

TONCHEV Plamen \& DaVARINou Polyxeni (2017), Chinese Investment in Greece and the Big Picture of Sino-Greek Relations, Athènes : Institute of International Economic Relations, <http://idos.gr/wpcontent/uploads/2017/12/Chinese-Investment-in-Greece_4-12-2017.pdf>.

Tonchev Plamen [dir.], Bentis Angelos, Carulas Caroline, Mihalaris Chris, PAPOUTSAs George (2018), China's Image in Greece 2008-2018, Athènes : Institute of International Economic Relations, <http://idos.gr/wp-content/uploads/2018/10/China-Image-in-Greece_9-10-2018.pdf>.

TRAGRANIDES Calliopi (2018), Le partenariat stratégique sino-grec dans le cadre de la Route de la soie maritime du XXI e siècle (mémoire de master 1, Études chinoises), Université Paris Diderot, Paris.

Tzogopoulos George N. (2015), « China bought Greek sovereign bonds worth of $€ 6 \mathrm{bn}$ at the beginning of crisis ", en ligne sur China and Greece : <http://chinaandgreece.com/china-boughtgreek-sovereign-bonds-worth-6bn-euros-beginning-crisis/> (9 janvier 2019).

VAN DER PUTTEN Frans-Paul (2014), Chinese Investment in the Port of Piraeus, Greece: The Relevance for the EU and the Netherlands, La Hague : Clingendael, Netherlands Institute of International Relations, <https://www.clingendael.org/sites/default/files/pdfs/2014\%20\%20Chinese\%20investment\%20in\%20Piraeus\%20-\%20Clingendael\%20Report.pdf>. VAN DER PutTen Frans-Paul \& Meijnders Minke (2015), « China, Europe and the Maritime Silk Road », en ligne sur Clingendael, Netherlands Institute of International Relations : <https:// www.clingendael.org/publication/china-europe-and-maritime-silk-road> ( 9 janvier 2019).

WAKIM Nabil (2018), « Comment la Chine achète l'Europe de l'énergie », en ligne sur Le Monde : <https://www.lemonde.fr/economie/article/2018/08/29/la-chine-achete-l-europe-de-lenergie_5347305_3234.html>.

WORLD SHIPPING COUNCIL (2019), Top 50 World Container Ports, en ligne sur <http:// www.worldshipping.org/about-the-industry/global-trade/top-50-world-container-ports> (9 janvier 2019). 
XINHUANET (2017), Vision for Maritime Cooperation under the Belt and Road Initiative, en ligne sur <http://www.xinhuanet.com/english/2017-06/20/c_136380414.htm> (8 janvier 2019).

XINHUANET (2018), China, Greece ink MOU to promote Belt and Road Initiative, en ligne sur <http:// www.xinhuanet.com/english/2018-08/28/c_137425525.htm> (8 janvier 2019).

ZHAO Lei (2015), « New rules mean ships can be used by military », en ligne sur China Daily: <http://www.chinadaily.com.cn/china/2015-06/18/content_21036944.htm> (8 janvier 2019).

ZAND Bernhard (2018), « China's High Seas Ambitions », en ligne sur Spiegel Online : <http:// www.spiegel.de/international/world/china-increasing-overseas-ambitions-with-maritime-silkroad-a-1110735.html> (8 janvier 2019).

\section{NOTES}

1. En anglais, l'abréviation est $O B O R$, One Belt, One Road, plus tard appelée la Belt and Road Initiative (BRI). Le site gouvernemental chinois est le Belt and Road Portal ; Le site de la HSBC suit aussi l'actualité de la BRI.

2. «China's investment in influence: the future of $16+1$ cooperation », China News Analysis, ECFR, décembre 2016.

\section{RÉSUMÉS}

La nouvelle route de la soie, ou « Belt and Road Initiative » marque la fin de la diplomatie à profil bas de la Chine, au profit d'une expansion de projets tous azimuts qui concerne l'ensemble des pays du monde, mis à part l'Amérique du Nord. L'axe ferroviaire Europe-Asie est beaucoup moins important commercialement que l'axe portuaire liant la Chine, l'Asie du Sud-Est et du Sud, l'Afrique et l'Europe. La Méditerranée est devenue le cœur de cet axe, et la Grèce, sa tête de pont, dans le cadre de l'acquisition du port du Pirée par la compagnie chinoise cosco, troisième transporteur mondial. Cet investissement s'est traduit par une augmentation très nette du trafic maritime du Pirée, classé à partir de 2013 troisième port de conteneur de la Méditerranée. Les investissements chinois se sont par ailleurs considérablement développés dans l'énergie, les télécommunications, l'immobilier et le tourisme, à l'occasion de la crise de la dette grecque et des politiques de privatisations prônées par l'Union Européenne. Le poids économique de la Chine se traduit déjà par des dividendes politiques, la Grèce ayant signé, contrairement aux autres pays de l'UE, un protocole d'accord sur la «Belt and Road Initiative", et refusé de s'associer à une déclaration européenne sur la dispute issue de l'annexion d'ilots en Mer de Chine du Sud (2016), ou à une résolution de l'Europe concernant le bilan des droits de l'homme en Chine présentée à la Commission des droits de l'homme des Nations Unies à Genève (2017).

The New Silk Road or "Belt and Road Initiative" marks the end of the Chinese low-profile diplomacy, and a world wide expansion of investment projetcs which concerns all the continents, expect North America. The railway link between China and Europe is much less important for commerce that the Chinese development of ports in South East Asia, South Asia, Africa and Europe. The Mediterranean Sea is the main transit centre of this new axis, and Greece has 
become its pivot, with the acquisition by $\cos \mathrm{CO}$, the world's third maritime transport company of the Piraeus Port in Athens. Following this investment, Piraeus has raised to the third position of the container ports in the Mediterranean Sea. With Greece's debt crisis, and politics of privatisations launched by the European Union, Chinese investments have also diversified in energy, telecommunications, real estate and tourism. The economic influence of china finds expression in political dividends: contrary to other EU states, Greece has signed a memorandum of understanding with China on the Belt and Road Intitiative, and has refused to associate to an European statement on the South China Sea (2016) or to a European resolution on the assesment of human rights in China before the UN Commission of Human Rights in Geneva (2017).

INDEX

Mots-clés : nouvelle route de la soie, Belt and Road Initiative, Méditerranée, Grèce, Pirée, investissements chinois, influence chinoise, Union Européenne

Keywords : New Silk Road, Belt and Road Initiative, Mediterranean Sea, Greece, Piraeus, Chinese Investments, Chinese influence, European Union

\section{AUTEUR}

\section{GUILHEM FABRE}

Université Paul Valéry, Montpellier 3 - LLACS 